\title{
Comprehension of concrete and abstract words in semantic variant primary progressive aphasia and Alzheimer's disease: a behavioral and neuroimaging study
}

Sven Joubert ${ }^{1,2}$, Guillaume T. Vallet ${ }^{1,2}$, Maxime Montembeault ${ }^{1,2}$, Mariem Boukadi ${ }^{1,2}$, Maximiliano A.Wilson ${ }^{3,4}$, Robert Jr. Laforce ${ }^{5,6}$ Isabelle Rouleau ${ }^{7,8}$, Simona M. Brambati ${ }^{1,2}$

1 Département de psychologie, Université de Montréal, Montréal, QC, Canada 2 Centre de recherche Institut universitaire de gériatrie de Montréal (CRIUGM), Montréal, QC, Canada 3 Centre de recherche de l'Institut universitaire en santé mentale de Québec (CRIUSMQ) 4 Département de réadaptation, Université Laval, Québec, QC, Canada

5 Clinique Interdisciplinaire de Mémoire (CIME), CHU de Québec, QC, Canada 6. Département des Sciences Neurologiques, Université Laval, QC, Canada 7 Département de psychologie, Université du Québec à Montréal, Montréal, QC, Canada 8 Centre de recherche du Centre hospitalier de l'Université de Montréal (CHUM), Montréal, QC, Canada

Key words: concrete words, abstract words, semantic memory, conceptual knowledge, semantic variant primary progressive aphasia (svPPA), Alzheimer's disease (AD)

Corresponding author: Sven Joubert, Département de psychologie, Faculté des arts et des sciences, Université de Montréal, Montréal, QC, Canada.

E-mail: sven.joubert@umontreal.ca

Number of words: 6524

Short title (running head): comprehension of concrete and abstract words in svPPA and AD 


\begin{abstract}
The aim of this study was to investigate the comprehension of concrete, abstract and abstract emotional words in semantic variant primary progressive aphasia (svPPA), Alzheimer's disease (AD), and healthy elderly adults (HE) Three groups of participants (9 svPPA, 12 AD, 11 HE) underwent a general neuropsychological assessment, a similarity judgment task, and structural brain MRI. The three types of words were processed similarly in the group of AD participants. In contrast, patients in the svPPA group were significantly more impaired at processing concrete words than abstract words, while comprehension of abstract emotional words was in between. VBM analyses showed that comprehension of concrete words relative to abstract words was significantly correlated with atrophy in the left anterior temporal lobe. These results support the view that concrete words are disproportionately impaired in svPPA, and that concrete and abstract words may rely upon partly dissociable brain regions.
\end{abstract}




\section{Introduction}

Semantic variant primary progressive aphasia (svPPA) is a neurodegenerative disease characterized by progressive breakdown of semantic knowledge (Hodges \& Patterson, 2007; Hodges, Patterson, Oxbury, \& Funnell, 1992). The most recent clinical diagnosis criteria include impaired naming, impaired word comprehension, impaired object knowledge, and surface dyslexia/dysgraphia, while repetition and speech production are spared (Gorno-Tempini et al., 2011). This syndrome is associated with bilateral atrophy of the anterior temporal lobes (ATL), typically predominating in the left hemisphere (Hodges et al., 1992).

The nature of semantic memory breakdown in svPPA has been the focus of much research in recent years, but there is still debate concerning some of the specific deficits that characterize the semantic impairment in svPPA. One of those debates concerns the comprehension of abstract and concrete concepts. Concrete concepts are tangible entities that can be experienced via our senses and are typically highly imageable (e.g. objects). They are highly dependent upon the visual modality but also on other sensory modalities. Conversely, abstract concepts can be expressed only via language and are usually poorly imageable. A robust phenomenon known as the concreteness effect reflects an advantage in processing for concrete words (e.g. tomato, computer, shoe) over abstract words (e.g. liberty, belief, trust), both in terms of accuracy and reaction times in neurologically-intact participants (Paivio, 1991; Xiao, Zhao, Zhang, \& Guo, 2012). The disadvantage in processing abstract words has also been found to be enhanced in patients suffering from neurological conditions such as acquired deep dyslexia and dysgraphia and Alzheimer's disease (AD) (Franklin, Howard, \& Patterson, 1994, 1995; Peters, Majerus, De Baerdemaeker, Salmon, \& Collette, 2009). According to the classical dualcoding hypothesis (Paivio, 1986, 1991), concrete words are better processed because they benefit from both visual and verbal coding, while abstract words benefit only from verbal coding (but see Kousta, Vigliocco, Vinson, Andrews, \& Del Campo, 2011; Paivio, 2013, for a more recent debate). Thus, even though both concrete and abstract concepts can be expressed via language, concrete words have the additional advantage of being highly imageable and having richer semantic representations (Paivio, 1986, 1991). Alternatively, it has been proposed that abstract and concrete conceptual knowledge relies on qualitatively different representations, as abstract concepts, but not concrete concepts, are represented in an associative neural network (Crutch \& Warrington, 2005, 2010). Lastly, the Context Availability Theory attributes concreteness effects to a less detailed representation in memory for abstract than concrete concepts (Schwanenflugel, Harnishfeger, \& Stowe, 1988; Schwanenflugel \& Shoben, 1983). 
A number of studies have documented the reverse pattern in concrete and abstract word processing in svPPA patients. A number of single case studies and brief series have shown that svPPA patients were more impaired at processing concrete words than abstract words (Bonner, Ash, \& Grossman, 2010; Breedin, Saffran, \& Coslett, 1994; Catricala, Della Rosa, Plebani, Vigliocco, \& Cappa, 2014; Macoir, 2009; Papagno, Fogliata, Catricala, \& Miniussi, 2009; A. Reilly, Cross, Troiani, \& Grossman, 2007; Warrington, 1975; Yi, Moore, \& Grossman, 2007), an effect sometimes called reversal of the concreteness effect (RCE). This effect has been interpreted by some authors as resulting from atrophy to the inferior temporal cortex affecting visual association cortex and leading to the deterioration of visuoperceptual knowledge about objects (Yi et al., 2007). According to this view, RCE would result from a greater impairment in processing concrete words due to their imageability and crucial reliance on visual sensory knowledge (Bright, Moss, Longe, Stamatakis, \& Tyler, 2007) while abstract words rely more on verbal associations.

Other studies, however, did not find this reversal of concreteness in svPPA (Hoffman, Jones, \& Lambon Ralph, 2013; Hoffman \& Lambon Ralph, 2011; Jefferies, Patterson, Jones, \& Lambon Ralph, 2009). Authors who have not found RCE in svPPA suggested that when studying abstract and concrete word processing, studies should strictly control for factors such as word frequency and imageability of stimuli used in the semantic tasks. Indeed, they suggested that the use of higher frequency words to investigate abstract knowledge and the lack of strict control of imageability may benefit abstract words and may have resulted in RCE. A synonym judgment task combining a large manipulation of concreteness (i.e. imageability) and good control of word frequency should reliably lead to better processing of concrete words over abstract words (Hoffman \& Lambon Ralph, 2011). These authors also suggested that premorbid level of functioning, such as occupation and education, may also account at least in part for RCE in the svPPA cases reported. In fact, several (but not all) of the previously reported cases of svPPA patients who showed RCE were professionals who presumably had a greater level of education and had developed more substantial abstract vocabulary, which may have helped these patients to better preserve their comprehension of abstract words (Hoffman \& Lambon Ralph, 2011; Macoir, 2009). Severity of the disease was also pointed out as a potential factor modulating the presence of RCE in svPPA patients. For instance, Macoir (2009) reported the longitudinal single-case study of patient SC who initially presented RCE and greater deficits for perceptual/structural knowledge, but with disease progression RCE vanished as well the distinction between visual perceptual and non-perceptual knowledge. It is thus possible that RCE may be present earlier in the 
disease process, and that studies that did not find this advantage for abstract words recruited patients who were at more advanced stages.

Another factor which has not been investigated in detail in previous studies is the impact of other components that might ground concepts. Particularly, abstract concepts have been showed to be more emotionally valenced than concrete concepts (Kousta et al., 2011). Nonetheless, no study seems to have tested whether emotional words, especially abstract words, are processed similarly to non-emotional words in svPPA. Studies in normal healthy participants have found that emotional valence was accessed earlier than semantic aspects such as concreteness of verbs (Palazova, Sommer, \& Schacht, 2013). Furthermore, one study showed that in AD, abstract words deteriorated more quickly than concrete words when there was no emotional valence to the words, but this difference between abstract and concrete words was not observed when emotional words were used (Giffard, Laisney, Desgranges, \& Eustache, 2015). This suggests that words may be less prone to deterioration in some neurodegenerative diseases when they are emotionally anchored. It remains to be explored whether this is the case in SVPPA patients.

Little is known about the neuroanatomical bases of abstract and concrete word processing in neurodegenerative diseases. Neuroimaging was reported in some case reports but only showed the pattern of atrophy, without allowing to correlate the behavioral data with RCE (Macoir, 2009; Papagno, Capasso, \& Miceli, 2009; A. Reilly et al., 2007). Some studies found RCE in svPPA and suggested that RCE may result from underlying damage to the inferior temporal cortex causing a breakdown of structural and perceptual knowledge about concrete concepts (Macoir, 2009; Yi et al., 2007). This is supported by a study that measured cortical thickness in a subgroup of 5 svPPA patients, who showed an advantage in processing abstract verbs relative to concrete verbs, and which revealed significant cortical thinning in visual association areas within the anterior, lateral and ventral portions of the temporal lobes, while greater difficulty for concrete verbs relative to abstract words was related to thinning of the right anterior temporal cortex (Bonner et al., 2009). To our knowledge, only one recent study investigated the neural correlates of concrete and abstract word processing in a group of svPPA (Cousins, York, Bauer, \& Grossman, 2016). This study, which also found RCE in svPPA, showed that the effect was associated with atrophy in the left ATL in svPPA, while a typical CE in the behavioral variant of Frontotemporal dementia (bv-FTD) was associated with bilateral atrophy in the inferior frontal cortex. According to the initial "hub-and-spoke" theory of semantic memory, conceptual representations emerge from the interaction of modality-specific association cortices with a transmodal hub located in the ATL bilaterally (Patterson, Nestor, \& Rogers, 2007). In a recent version of this 
model (Ralph, Jefferies, Patterson, \& Rogers, 2017), the notion of a "graded ATL semantic hub" emerged, based on a decade of original work in this field. According to this model, a cross-modal hub is centered on the ventrolateral ATL, while semantic function varies in a graded manner across specific ATL subregions within the broader ATL. Notably, the medial ATL responds more to visual or concrete concepts due to greater connectivity to visual systems (relative to auditory or linguistic systems), whereas the anterior STS-STG responds more to abstract concepts because of its greater connectivity to language systems (Ralph et al., 2017).

Neuroimaging studies have also contributed to our understanding of concrete and abstract word processing. In a meta-analysis of the neuroimaging literature on semantic memory, Binder et al. (Binder, Desai, Graves, \& Conant, 2009) reported that abstract concepts were found to activate the left inferior frontal gyrus (IFG), superior portions of the left ATL, and the superior temporal sulcus (STS). In contrast, concrete concepts were associated with more distributed activation across the two hemispheres, more specifically in bilateral angular gyrus (AG), bilateral dorsomedial prefrontal cortex, left posterior cingulate and left fusiform cortex. In another neuroimaging meta-analysis (Wang, Conder, Blitzer, \& Shinkareva, 2010), abstract concepts were associated with activity in the left IFG and the left temporal pole (TP), while concrete concepts were associated with activity in the left AG, left posterior inferior temporal cortex, and posterior anterior cingulate. Finally, a recent study investigated processing of abstract and concrete words by using both fMRI connectivity in healthy subjects and a behavioral study of 10 aphasic stroke patients with left temporoparietal lesions (SkipperKallal, Mirman, \& Olson, 2015). Results from this study suggest (partially) functionally distinct networks for abstract and concrete words. Indeed, in the fMRI study the anterior IFG was functionally associated with the angular gyrus and posterior STS during abstract word processing, while the aIFG was functionally connected to the MTG and TP during concrete word processing. The lesion study also showed that patients with lesions in the left temporoparietal cortex (TPC), including the AG, had specific difficulty discerning the meaning of abstract words but not concrete words. This study thus suggests a key role of the left TPC in comprehension of abstract concepts.

Clearly, more neuroimaging studies are needed to better understand the neuroanatomical bases of concrete and abstract word processing. Our objective is to achieve this goal with a group of svPPA patients. More specifically, the aims of the present study were: 1) to investigate comprehension of concrete and abstract words in a group of svPPA patients using a similarity judgment task, and to compare their performance with a group of $\mathrm{AD}$ patients and healthy controls matched for age and education; 2) to investigate the role of emotional anchorage of abstract words by comparing 
comprehension of abstract emotional and non-emotional words; and 3) to test the association between brain atrophy and performance in the task using Voxel-Based Morphometry (VBM). The hypothesis was that svPPA patients would present with a reversal of the concreteness effect relative to the degree of anchorage of concepts (abstract > emotional > concrete), while AD patients would present with an enhancement of the concreteness effect. It was also hypothesized that RCE in svPPA would correlate with atrophy in the ATL. Specific attention was paid to a number of factors that have previously been pointed out as potential confounds. First of all, svPPA and AD patients were in a mild stage of the disease, thus controlling for disease severity. Secondly, patients and participants were matched for age and level of education, thus avoiding potential differences in terms of premorbid experience between groups.

\section{Material and methods}

\subsection{Participants}

Nine patients with a clinical diagnosis of semantic svPPA ( 2 women, 7 men), 12 patients with AD (4 women, $8 \mathrm{men}$ ), and 11 healthy elderly adults (HE) (4 women, 7 men) took part in this study. Demographics of participants are presented in Table 1. The three groups were matched for age, education, and gender (see Table 1). The svPPA and AD patients were recruited through La Clinique interdisciplinaire de Mémoire du Centre hospitalier universitaire (CHU) de Québec and referred by a neurologist with expertise in neurodegenerative diseases (RL). SvPPA patients were diagnosed according to current criteria (Gorno-Tempini et al., 2011). Diagnosis of AD was made based on the research criteria of the National Institute of Neurological and Communicative Disorders and StrokeAlzheimer's Disease and Related Disorders Association (McKhann et al., 2011) and the clinical criteria for dementia of the Alzheimer type (American Psychiatric Association, 1994). General exclusion criteria were: first spoken language other than French, left-handedness, developmental learning disabilities, past psychiatric disorder, history of traumatic brain injury, and uncorrected hearing and vision problems. The study was approved by the research ethics committee of the CHU de Québec (Project \#2015-1909) and written informed consent was obtained from all participants. 
Table 1: Demographic and neuropsychological data for the healthy elderly (HE), patients with Alzheimer's disease (AD) and semantic variant primary progressive aphasia (svPPA).

\section{HE $(n=11) \quad$ AD $(n=12) \quad$ Sv-PPA $(n=9) \quad p$ value Group comparison}

Demographic

$\begin{array}{rccccc}\text { Age } & 67.0(9.1) & 70.42(8.3) & 65.19(11.2) & 0.44 & \mathrm{HE}=\mathrm{AD}=\mathrm{SD} \\ \text { Sex }(\mathrm{M} / \mathrm{F}) & 7 / 4 & 8 / 4 & 7 / 2 & 0.78 & \mathrm{HE}=\mathrm{AD}=\mathrm{SD} \\ \text { tion (years) } & 17.36(3.2) & 15(3.9) & 16.11(4.1) & 0.47 & \mathrm{HE}=\mathrm{AD}=\mathrm{SD}\end{array}$

\section{Global}

Education (years) $17.36(3.2) \quad 15(3.9)$

MMSE $29.09(0.7) \quad 25.25(2.7)$

$25.22(2.1)$

$<0.001 * * *$

$\mathrm{HE}>\mathrm{SD}=\mathrm{AD}$

Visual perception

\section{Benton Faces \\ Benton Lines \\ $47.82(3.0)$ \\ $27.73(2.3)$}

Visuospatial perception

Rey-Osterrieth (copy)

Clock drawing (on command)

Clock drawing (copy)

\section{Executive Functions}

$\begin{array}{cc}\text { Stroop WC }(\mathrm{sec}) & 130.64(30.1) \\ \text { TMT A }(\mathrm{sec}) & 31.18(4.2) \\ \text { TMT B }(\mathrm{sec}) & 63.82(19.2)\end{array}$

\section{Working memory}

Digit span (forward)

Digit span (backward)

Digit span (total)

\section{Episodic Memory}

RAVLT 1-5
RAVLT (delayed free
recall)
RAVLT (delayed rec)
Rey-Osterrieth (delayed
recall)

$$
45.17 \text { (3.2) }
$$$$
24.67 \text { (6.9) }
$$

$32.55(2.7)$

$$
9.36 \text { (1) }
$$

$9.68(0.5)$

\section{$27.33(7.9)$ \\ 7.46 (2.4) \\ $9.25(0.8)$}

$\begin{array}{ll}44.44(3.4) & 0.05 \\ 26.56(2.4) & 0.28\end{array}$

$29.67(4.5)$

7.78 (1.9)

$9.72(0.4)$
$135.12(35.8)$

47.89 (13)

$113.44(66.8)$

$<0.01^{* *}$
0.12
$<0.001^{* * *}$

$18.73(4.6)$

$52.73(7.4)$

$10.64(2.7)$

46.73 (2.2)

$20.64(4.7)$
$9.11(2.1)$

$5.44(2)$

$14.56(3.7)$

$$
\begin{gathered}
9.83(1.3) \\
6(2) \\
15.83(2.6)
\end{gathered}
$$

$28.42(5.8)$

$$
2 \text { (2.9) }
$$

$32.75(7.9)$

$5.92(4.6)$

$\begin{array}{cc}29.5(7.8) & <0.001 * * * \\ 4.67(2.7) & <0.001 * * * \\ 41(5.7) & <0.001 * * * \\ 8.44(5.2) & <0.001 * * *\end{array}$

$8.44(5.2)$

$$
12.67(8.7)
$$

31.67 (12.4)

$9.11(7.8)$

$13.22(5.7)$

30.67 (12)

$<0.001 * * *$
0.07

0.11

$0.03 *$

$48.42(2.1)$

$14.42(7.1)$

$20.50(7.9)$

40.83 (13.7)

$$
\begin{aligned}
& \mathrm{HE}>\mathrm{SD}=\mathrm{AD} \\
& \mathrm{HE}=\mathrm{AD}=\mathrm{SD} \\
& \mathrm{HE}=\mathrm{AD}=\mathrm{SD} \\
& \mathrm{HE}>\mathrm{AD}=\mathrm{SD} \\
& \mathrm{HE}=\mathrm{AD}=\mathrm{SD}
\end{aligned}
$$

$\mathrm{SD}=\mathrm{HE}<\mathrm{AD}$

$\mathrm{HE}=\mathrm{AD}=\mathrm{SD}$

$\mathrm{SD}=\mathrm{HE}<\mathrm{AD}$

Free fluency $66.09(17.7)$

QVA with the

otes. $p=p$ values computed for each ANOVA with the group (HE, AD, SD) as a between subject variable (or chi square test for gender); $*=p<0.05 ; * *=p<0.01 ; * * *=p<0.001 ;$ MMSE= Mini-Mental State Examination; $T M T=$ Trail Making Test; $R A V L T=$ Rey Auditory Verbal Learning Test; $B N T=$ Boston Naming Test; $P P T T=$ Pyramids and Palm Trees Test. 


\subsection{Neuropsychological assessment}

All participants completed a standard battery of neuropsychological tests. It assessed general cognitive status by means of the MMSE (Folstein, Folstein, \& McHugh, 1975), as well as more specific cognitive domains. These domains included nonverbal and verbal episodic memory (Meyers \& Meyers, 1995; Immediate and differed recall of the Rey Complex Figure Test, Osterrieth, 1944; Rey Auditory Verbal Learning Test, Rey, 1960), language and semantic memory (Boston Naming Test, Kaplan, Goodglass,

\& Weintraub, 1983; Pyramids and Palm Trees Test, Howard \& Patterson, 1992); free, letter and semantic fluency (Protocole MEC, Joanette, Ska, \& Côté, 2004), working memory (Forward and Backward Digit-span, Wechsler, 1997), visual perception (Benton Line Orientation test, Benton, Varney, \& Hamsher, 1978; Benton Facial Recognition, Benton \& Van Allen, 1968; Qualls, Bliwise, \& Stringer, 2000), visuoconstructional skills (Meyers \& Meyers, 1995; copy of the Rey Complex Figure Test, Osterrieth, 1944); Clock-drawing Test (Rouleau, Salmon, Butters, Kennedy, \& McGuire, 1992), and executive functions (Stroop-Victoria Test, Strauss, Sherman, \& Spreen, 2006; Trail making test A\&B, Tombaugh, 2004). Results are presented in Table 1.

\subsection{Similarity judgment task}

Semantic knowledge of concrete, emotional and abstract words was assessed in a written semantic similarity judgment task. Participants completed a total of 30 trials with 10 stimuli per condition (10 concrete words, 10 emotional abstract words and 10 abstract words). Each trial was composed of triplets of words: two nouns with a very similar meaning and a third semantically irrelevant noun. All words were matched for lexical frequency $(F<1)$. Concrete nouns and emotional/ abstract nouns showed high (6.36) and low (Abstract and Abstract-emotional = 2.9) imageability scores respectively, considering that concrete words are easily imageable contrary to abstract words (Breedin et al., 1994; Macoir, 2009). It can be assumed that words with high imageability values exert greater demands on sensory and perceptual levels of processing, while the words with low imageability values exert greater demands on more abstract conceptual processing (Martensson, Roll, Apt, \& Horne, 2011). Lexical frequency of words was taken from the French Lexical Database Lexique 3.01 (New, Pallier, Brysbaert, \& Ferrand, 2004; http://www.lexique.org/) while word imageability was taken from the University of Ottawa OMNILEX database (OMNILEX: A Computerized Database on the French Lexicon; http://www.omnilex.uottawa.ca/; Desrochers, 2006). 


\subsubsection{Procedure}

The experiment was designed and ran using OpenSesame 2.91 (Mathot, Schreij, \& Theeuwes, 2012). The experiment started with a written and oral presentation of the instructions. Participants had to determine which word among the visually-presented triplet was the least similar from the two other semantically related nouns. In other words, participants had to find the intruder among the three displayed words.

Each trial started with a fixation point displayed in the middle of the screen for $500 \mathrm{~ms}$. Participants were then simultaneously presented with three written nouns aligned horizontally in the center of the screen. The triplet remained on the screen until a response was provided.

The position of the nouns on the screen was pseudo-randomized so that the correct response was equally distributed across the three possible positions (left, center, right). Trial order was randomly determined for each participant. Each word occurred only once during the experiment.

\subsubsection{Statistical analyses}

Analyses were conducted on correct response rates (CR) computed in each condition since participants have no specific time-related instructions. Analyses of variance (ANOVA) were ran with the Group (HE vs. AD vs. svPPA) as between-subjects variable and the Item-Type (concrete vs. emotional vs. abstract) as within-subjects variable. Then separate ANOVA were computed, one for each group, with the Item-Type as within-subjects variable. Homogeneity of variance (i.e. sphericity in a repeated measures design) was assessed using the Mauchly's Test for sphericity. When sphericity was violated, the Greenhouse-Geisser correction was applied and reported in the results. Posthoc analyses were computed using unilateral Student's t-test corrected for multiple comparisons using the Holmes procedure. A $p$ value (corrected when needed) inferior to 0.05 was used as a significant threshold for all analyses.

\subsection{Voxel-Based Morphometry}

\subsubsection{Image acquisition}

Nine svPPA patients, $11 \mathrm{AD}$ patients and $10 \mathrm{HE}$ who completed the similarity judgment test underwent a Magnetic Resonance Imaging protocol including a high-definition T1 brain image. The brain structural MRI scans were obtained with a 3T Philips Achieva TX scanner at IRM Québec-Mailloux in Quebec City. A volumetric magnetization prepared rapid gradient echo (MP-RAGE) sequence was used to acquire a high-resolution $\mathrm{T} 13 \mathrm{D}$ structural image $(\mathrm{TR}=8.2 \mathrm{~ms}, \mathrm{TE}=3.7 \mathrm{~ms}, \mathrm{FoV}=250 \mathrm{~mm}$, 
flip angle $=8 \circ, 256 \times 256$ matrix, 180 slices/volume, slice thickness $=1 \mathrm{~mm}$, no gap).

\subsubsection{Data analysis}

Image preprocessing. The structural images were preprocessed using voxel-based morphometry (VBM) implemented in SPM12 using MATLAB 7.14.0.739 (Mathworks, Natick, MA). The images were segmented into gray (GM) and white (WM) matter. Affine registered tissue segments were used to create a custom template using the DARTEL (diffeomorphic anatomical registration using exponentiated lie algebra) approach (Ashburner, 2007). For each participant, the flow fields were calculated during a template creation, which described the transformation from each native GM image to the template. These were then applied to each participant's GM image. The VBM analysis was based on modulated GM images, whereby the GM value for each voxel was multiplied by the Jacobian determinant derived from spatial normalization to preserve the total amount of GM from the original images (Ashburner \& Friston, 2000). The resulting modulated and normalized images were then smoothed with a Gaussian kernel of 8 mm FWHM.

\subsubsection{Statistical analysis}

Accuracy on the semantic similarity judgment task with concrete and abstract words was entered in a

single multiple regression statistical model as separate covariates of interest. Smoothed GM images of all participants were entered as a single group in the statistical model. Age and gender were entered as nuisance covariates. Specific contrasts were set in order to identify the brain regions that correlated with concrete and abstract word accuracy, respectively. The correlation was tested using a [1] $t$ contrast, assuming that decreased semantic processing would be associated with decreased GM volumes. The significance of each effect of interest was determined using the theory of Gaussian fields (Friston et al., 1995). Statistical threshold of $p<0.001$ uncorrected for multiple comparisons was used. In order to characterize the pattern of atrophy in patient groups, two separate two-sample T-test models were used to compare GM volume in AD versus controls and in SvPPA versus control (Figure 2).

\section{Results}

\subsection{Semantic similarity judgment task}

The ANOVA performed on the CR revealed a main effect of Group, $F(2,29)=14.83, p<.05$, $\eta^{2} g=.39$, whereby $\mathrm{HE}(\mathrm{M}=0.9, \mathrm{SD}=0.3)$ performed better than $\mathrm{AD}$ patients $(\mathrm{M}=0.81, \mathrm{SD}=0.4)$, who in turn performed better than svPPA patients $(0.6 \pm 0.5)($ all $p<.05)$. 
The Item-Type factor was also significant, $F(1.61,46.73)=6.54, p<.05$. A significant difference was observed between concrete words and both emotional and abstract words $(p<.05)$, whereas no significant difference was observed between the emotional and the abstract words $(p=0.91)$. Finally, the Group by Item-Type interaction was not significant, $F(3.22,46.73)<1$.

Following our a priori hypothesis about the Group by Item-Type interaction, we conducted separate ANOVA for each group on Item-Type as the within-subjects variable (see Figure 1) and $a$ priori pairewise $t$ tests of Student were used to further analysed the data for each group (see Howell, 2012). In the HE group, the ANOVA revealed a significant effect of Item-Type, $F(2,20)=3.92$,

$p<.05, \eta^{2} g=.2$. Emotional words tended to be better processed than concrete words $(p=0.05)$, whereas no other significant difference was observed $(p=0.91)$. On the contrary, the Item-Type factor was not significant in the AD group, $F(2,22)=2.05, p=.15, \eta^{2} g=.04$ (no significant difference between the conditions, $\mathrm{p}>0.50)$ or in the svPPA group, $F(2,16)=2.2, p=.14, \eta^{2} g=.10$. However in the svPPA group, abstract nouns were processed significantly better than concrete nouns $(p<.05)$, whereas processing emotional nouns was not statistically different from processing of $\operatorname{abstract}(p=0.41)$ and concrete nouns $(p=0.28)$.

\subsection{Voxel-Based Morphometry Results}

A positive correlation between concrete word semantic judgment and GM volume was observed in the left anterior temporal lobe, both medial $(\mathrm{x}=-29, \mathrm{y}=-3, \mathrm{z}=-30, \mathrm{~T}=4.37 ; \mathrm{x}=-35, \mathrm{y}=-21, \mathrm{z}=-32, \mathrm{~T}=3.55)$ and lateral portion $(x=-62, y=-12, z=-17, T=3.45)$. No significant correlation was observed between abstract word semantic judgment and the GM (see Figure 3). 
Figure 1. Correct response rates for healthy control (HE), patients with Alzheimer's disease (AD) and patients with the semantic variant of primary progressive aphasia (svPPA) across the experimental conditions: concrete nouns, (abstract) emotional nouns and abstract nouns. Bars represent standard errors corrected for within-subjects design (see Morey, 2008).

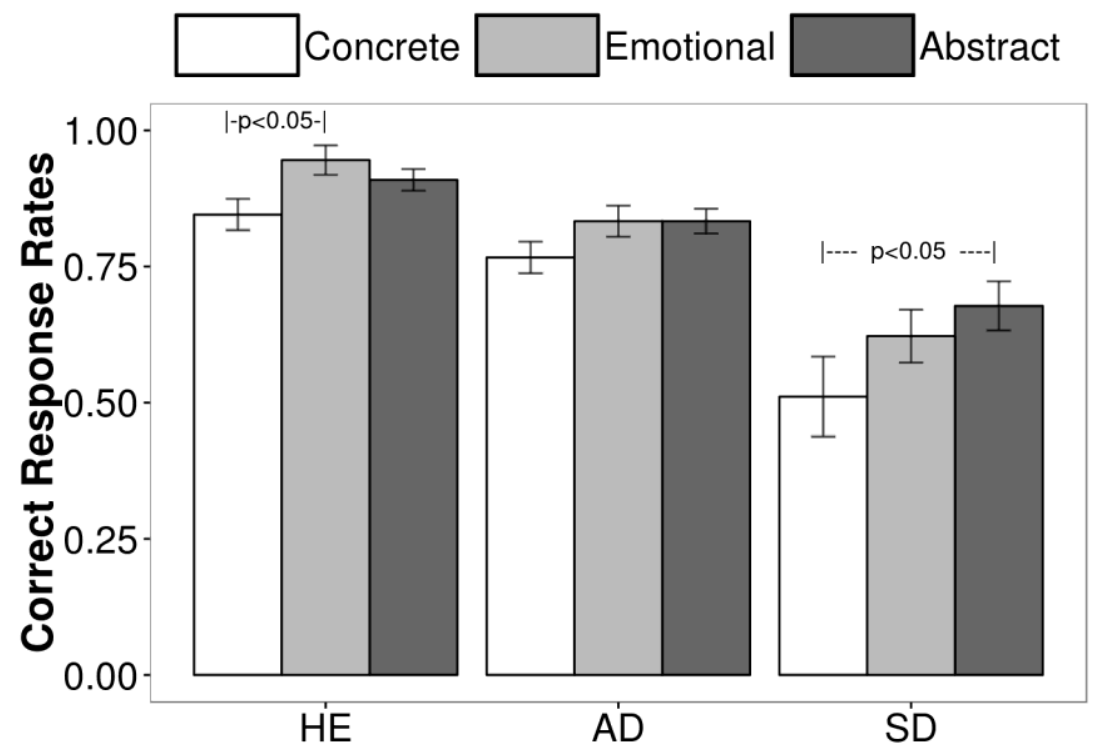

Figure 2. Patterns of atrophy of patients with (A) semantic variant primary progressive aphasia (svPPA versus controls), and (B) Alzheimer's disease (AD versus controls).
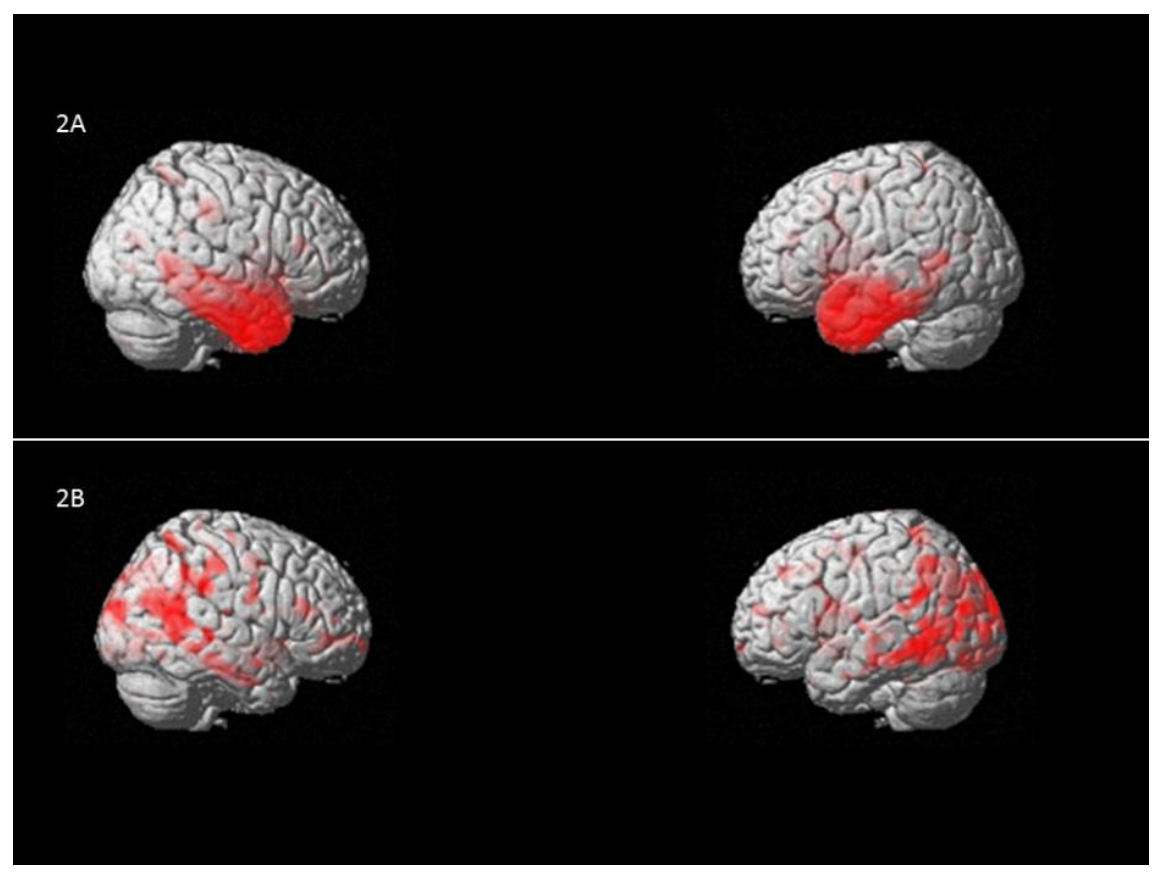
Figure 3. Positive correlation between concrete word semantic judgment and gray matter (GM) volume in the medial (A) and lateral (B) portions of the left anterior temporal lobe was observed.

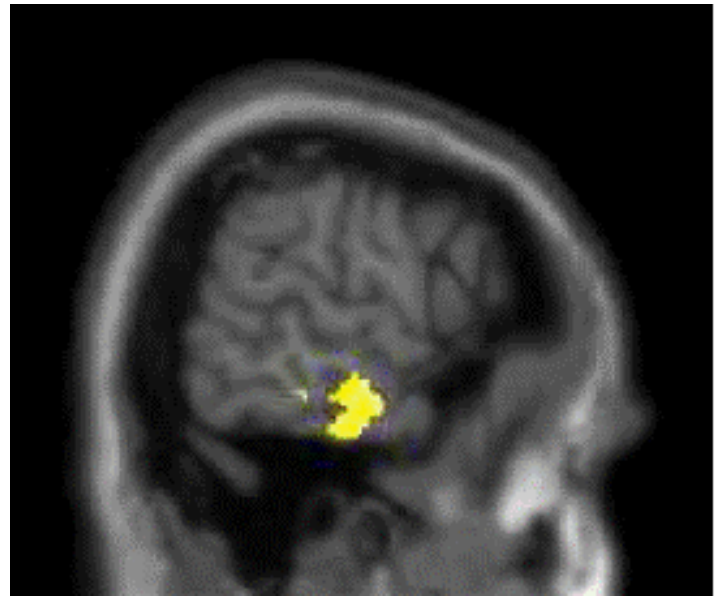

$X=-60$

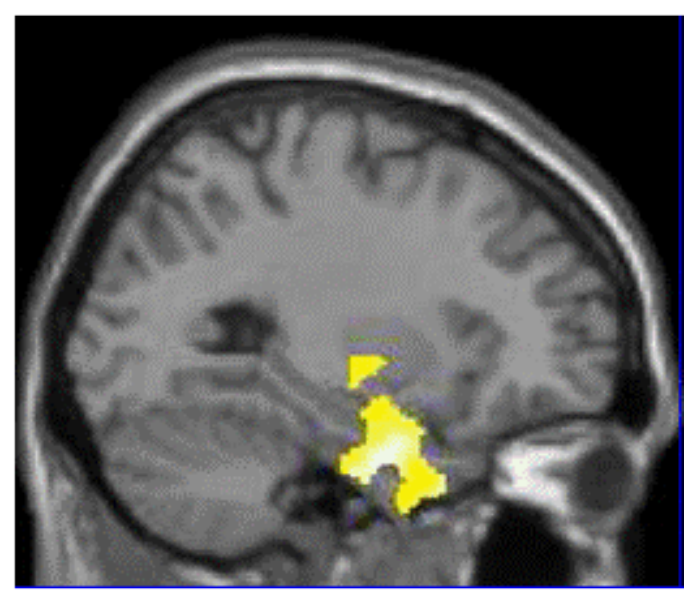

$X=-27$

\section{Discussion}

The aim of the present study was to investigate abstract, abstract emotional and concrete word processing in svPPA, AD, and normal aging. Behavioral results showed that the svPPA group was significantly more impaired at processing concrete words relative to abstract words in a similarity judgment task, while this effect was not found in the AD and control groups. Comprehension of emotional abstract words in svPPA was mid-way between that of concrete and abstract words. In the AD group, there was no significant difference between any word categories. In the HE group, abstract emotional words were better processed than concrete words. Finally, VBM analyses showed that concrete word comprehension correlated with the volume of grey matter in the left ATL region and the anterior hippocampus. In contrast, deficits in abstract word comprehension did not correlate with atrophy in any region.

Discrepancies found across studies regarding the RCE effect in svPPA may be explained in terms of insufficient control of factors such as word frequency and word concreteness/imageability. In the current study, a significantly greater impairment at processing concrete words relative to abstract words was found in svPPA, even though lexical frequency and concreteness of stimuli were controlled. Concrete, abstract, and abstract emotional word lists were matched for lexical frequency, and mean imageability values of concrete words were significantly greater than those of abstract and abstract emotional words. Therefore, our results corroborate those of former studies (Breedin et al., 1994; Catricala et al., 2014; Cousins et al., 2016; Macoir, 2009; Papagno, Capasso, et al., 2009; J. Reilly \& Kean, 2007; Yi et al., 2007) and suggest that the effect 
observed in svPPA is not due to confounding factors related to the properties of the stimuli (see also Catricala et al., 2014). Furthermore, abstract emotional and abstract words were matched for imageability, and despite this, svPPA patients exhibited a gradual decline inversely related to the anchorage of words. However, it is worth pointing out that in the current study concrete words were not better processed than abstract words by healthy controls and AD patients, contrary to what has been reported in previous studies (Paivio, 1991; Peters et al., 2009; Xiao et al., 2012). Although we do not have a clear explanation for this, one possibility is that our task was easy. Indeed, the effect is more typically found on reaction times during lexical decision. Similarly, mean frequency of triplets may have been higher than in previous studies, which may have resulted in higher scores and less differences in performance across word categories, but this is difficult to verify since frequencies are not the same across languages. It could also be argued that concrete triplets were simply more difficult than abstract triplets, and that patients were more impaired on the most difficult semantic condition, however this seems unlikely since word lists were matched for lexical frequency. Future studies could perhaps further investigate this question by using latent semantic analyses or measures of semantic distance between targets and distracters, if such measures are available. Regardless, comprehension of concrete words was significantly more impaired than that of abstract words only in the svPPA group. Differences in premorbid experience of abstract word processing (Hoffman \& Lambon Ralph, 2011), such as the level of educational achievement, are also unlikely to account for the results of the current study since all three groups were matched for number of years of education. Disease severity was also considered in the present study; both AD and svPPA patients were in a mild stage of the disease. The longitudinal single case study of patient SC (Macoir, 2009) showed that this patient initially showed RCE, but that the difference between concrete and abstract word processing faded with disease progression. This raises the possibility that RCE may be more present early in the disease. This needs to be further tested by means of longitudinal studies of svPPA patients.

The pattern of results found in svPPA is challenging to interpret in terms of Paivio's dual-coding hypothesis (Paivio, 1986, 1991), which posits that concrete words benefit from both visual and verbal coding while abstract words benefit only from verbal coding. Accordingly, concrete words should be more resistant to brain damage than abstract words. In contrast, the greater impairment for concrete words in svPPA is compatible with the sensory deficit hypothesis in svPPA. According to this view, the atrophy in the anterior temporal lobes leads to impaired high-level visual processing (see Barense, Rogers, Bussey, Saksida, \& Graham, 2010). The results in svPPA are also consistent with the differential structural framework theory (Crutch, 2006; Crutch, Connell, \& Warrington, 2009; Crutch, Troche, Reilly, \& Ridgway, 2013; Crutch \& Warrington, 2005, 2007, 2010), which suggests qualitatively different semantic representations and 
functional organization of concrete and abstract concepts. According to this view, abstract concepts are represented in an associative network while concrete concepts have a categorical organization, and these differences must be reflected in the neural bases of conceptual knowledge (Crutch \& Warrington, 2005). Abstract words are acquired in the context of language (Breedin et al., 1994), have richer associations and multiple meanings. Concrete concepts in contrast are categorically organized and processed according to their semantic similarities. Concepts of the same category can be compared based on their shared sensory, motor and functional features. Thus, greater impairment in svPPA for concrete concepts may reflect greater damage to this underlying categorical organization. It must be noted however that Crutch and Warrington (2005) insist on the relative rather than absolute distinction and non-mutually exclusive nature between the associative and categorical representational framework. Finally, the context availability theory suggests that abstract words have a less detailed representation in memory than concrete concepts due to less contextual information available (Schwanenflugel et al., 1988; Schwanenflugel \& Shoben, 1983). The authors reported that in healthy controls the advantage for concrete words disappeared when concrete and abstract words were presented within a context (e.g. sentences), as opposed to when they were presented as single words, suggesting that abstract words may rely to a greater extent on the context. Accordingly, the context of a sentence is more important in determining the meaning of an abstract word that has multiple meanings and whose meaning depends on the specific context in which it is presented. In contrast to the differential structural framework theory, which suggests a qualitatively different organization for concrete words rather than a quantitative one, results of the current study are more difficult to interpret in terms of the context availability theory, even though not entirely inconsistent. In fact, concrete and abstract words were presented as single words in this study and did not benefit from contextual information such as sentences. Thus, according to this view we should have expected a typical concreteness advantage in patients.

In regard to emotional processing, the svPPA group is the only group to exhibit performance for emotional abstract concepts at the mid-point between abstract and concrete nouns, whereas these nouns tend to be better processed in HE and no difference is observed in the AD group (see also, Catricala et al., 2014; Giffard et al., 2015). According to Kousta et al. (2011), abstract concepts are more grounded in emotional valence while concrete concepts are more grounded in sensori-motor components. Yet, emotional abstract words rely more on emotional valence than non-emotional abstract words, even when these two types of words are matched for imageability, frequency or other linguistic parameters. Therefore, the fact concrete words are less well processed than emotional abstract words, which in turn are less well processed than abstract words in svPPA patients, may suggest that degradation of knowledge in svPPA is inversely correlated to the anchorage of concepts (assuming sensorimotor components are a more important anchor 
than emotion). This assumption is also compatible with the evolution of brain atrophy in svPPA, starting from the anterior temporal lobes and extending with disease progression to the ventromedial frontal and the left anterior insular regions (Brambati et al., 2009) which are key structures in emotion processing (Phan, Wager, Taylor, \& Liberzon, 2002). Along the same line, specific socioemotional disturbances are encountered in this syndrome (Rosen et al., 2006) and their onset may mirror the progression of atrophy to frontal and insular regions. One difference between our study and work by Kousta et al. (2011) is that we used imageability as a proxy for concreteness (i.e. concrete words were highly imageable while abstract words were not), whereas Kousta et al. used concrete and abstract words that were matched on imageability. These methodological differences must be considered when comparing results.

Even though it is evident that concrete and abstract word comprehension relies on a common distributed neural network, it is possible that differences in the representations of abstract and concrete words may be underpinned by at least partly different neural regions, such as suggested by clinical dissociations (for review, see Shallice \& Cooper, 2013). For instance, aphasic patients with frontal or temporoparietal lesions have been found to present with specific difficulties in processing abstract words, while an anomic aphasic patient with occipital lesions exhibited difficulty in concrete word processing (Martensson et al., 2011; Tyler, Ostrin, Cooke, \& Moss, 1995). Other studies also showed that semantic aphasia patients who suffered prefrontal and/or temporoparietal infarction show deregulated semantic cognition across different modalities, i.e. they had difficulties in semantic tasks due to a failure of executive control (Corbett, Jefferies, \& Ralph, 2011; Jefferies \& Lambon Ralph, 2006). Abstract words, which are more associative and context-dependant, may thus be more reliant on these regions involved in semantic control processes. Concrete words however may depend to a greater extent on their sensorimotor features (Barsalou, 2008; Vallet, Simard, Versace, \& Mazza, 2013). It has been suggested, for example, that damage to inferotemporal cortex and visual association cortices may lead to degraded visual perceptual and structural features of objects, and that damage to these regions of the visual ventral stream may account for the disproportionate impairment of svPPA patients found in processing concrete concepts (Bonner et al., 2009; Macoir, 2009; Yi et al., 2007). Further evidence in favor of partly dissociable neuroanatomical substrates of abstract and concrete word processing comes from neuroimaging studies of healthy subjects. For instance, abstract word processing has been found to activate a subregion of the left inferior frontal gyrus more strongly than concrete words (BA 45), while the reverse contrast showed that specific activity for concrete words was observed in the left basal temporal cortex including the perirhinal cortex (Fiebach \& Friederici, 2004). 
Neuroimaging results in the current study showed that poorer concrete word comprehension in the synonym judgment task correlated with gray matter volume loss in the left anterior temporal lobe, more specifically in the temporopolar cortex, and the anterior section of the hippocampus (see Figure 3). These results are similar to those of Cousins et al. (2016). To the best of our knowledge, the4 latter study is the only other that investigated the relation between this dissociation in concrete and abstract word processing and gray matter atrophy in a group of svPPA patients. They showed RCE in svPPA patients, while bv-FTD patients showed a concreteness effect. Regression analyses showed that RCE was associated with left anterior-inferior temporal atrophy in SvPPA, while the CE was associated with bilateral inferior frontal atrophy in bv-FTD. In the current study, however, we used a different method whereby the svPPA and AD patients were entered in the statistical model as a single group in order to increase the brain atrophy variability in the brain and statistical power. The strength of this method it that it relies on the neuroanatomical variability of the different patient groups pooled in the same analysis (see for instance Amici et al., 2007; Brambati et al., 2006; Joubert et al., 2016). Despite these methodological differences, processing concrete words was also associated with gray matter volume in the left anterior temporal lobe: impaired concrete word comprehension was associated with greater atrophy in this region. In contrast, we did not find abstract word processing to be associated with atrophy in any brain region.

These results suggest that the left ATL may have a more prominent role in processing concrete words compared with abstract words. This is not consistent with the initial version of the hub and spokes model which suggests that all types of concepts, concrete and abstract, are processed likewise by the ATL region, which acts as a central, amodal hub of conceptual knowledge (Lambon Ralph, Cipolotti, Manes, \& Patterson, 2010; Patterson et al., 2007). According to this view, we should not expect differential involvement of the ATL region in processing concrete and abstract words. However, the most recent and updated version of this model (Ralph et al., 2017) seems to accommodate our results. The graded hub hypothesis suggests that that the ventrolateral ATL is the core of the hub, but that the function varies in a graded manner across adjacent ATL subregions, i.e. the latter subregions are more tied to a specific input modality. According to this model, the more medial ATL responds more to visual stimuli and concrete concepts, while the anterior superior temporal sulcus (aSTS) responds more to auditory stimuli, spoken words, and abstract concepts (Ralph et al., 2017).

Other authors also suggested that greater difficulties in processing concrete words in svPPA are associated with damage to visual association cortex of the temporal lobe causing a breakdown of structural and perceptual knowledge about concrete concepts (Bonner et al., 2009; Macoir, 2009; Yi et al., 2007). This view is consistent with the sensory-motor approach, according to which concrete concepts are represented 
under sensory-perceptual and action-motor features (Barsalou, 2008; Martin, 2007; Pulvermuller, 2005). Although the degraded visual feature knowledge view provides a solid account of the impairment for concrete concepts in svPPA, it does not offer in our view a complete account of the pattern of semantic impairment in svPPA. For instance, Bonner et al. (2009) indicate that most evidence for a semantic memory deficit in SD in fact comes from demonstrations of difficulty with visual-perceptual material. But in a clinical setting, svPPA patients are often unable to recognize man-made and biological objects regardless of the sensory modality in which they are presented. The semantic deficits are multimodal and not only limited to the visual modality.

In accordance with the hypothesis of degraded visual feature knowledge, significant cortical thinning has been reported in svPPA in visual association areas within the anterior temporal cortex, as well as lateral and ventral atrophy extending more posteriorly in the temporal lobe (Bonner et al., 2009). In the current study, impaired concrete word comprehension was associated with gray matter atrophy in the left anterior temporal cortex, but did not correlate with more posterior visual association areas. Similarly, in a recent study by our group, RCE was found in epileptic patients who had undergone selective surgical resection of the ATL, while patients who had undergone selective amygdalo-hippocampectomy resection did not show this distinction between concrete and abstracts words (Loiselle et al., 2012). The resection of ATL in these patients was also anterior, sparing posterior visual association regions. Although these patients are clearly different from svPPA patients, results suggest that damage to more posterior visual association cortices such as damaged in svPPA is not necessary to produce RCE, since these regions were spared in patients with selective ATL resection. These results along with those of the current study raise the possibility that damage to the ATL may be sufficient to cause a disproportionate impairment in processing concrete concepts and that lesions to more posterior visual association cortex may not be as critical. In addition, the temporopolar cortex (TPC), the most rostral portion of the temporal lobe, includes high order visual association cortical regions, extending more anteriorly than previously thought (Ding, Van Hoesen, Cassell, \& Poremba, 2009). In a recent study, RCE was associated with left anterior and inferior temporal atrophy in svPPA (Cousins et al., 2016). The authors suggested that concrete concepts may depend in part on sensory-motor features, with a significant role of the inferior temporal gyrus in visual feature knowledge and the superior temporal gyrus in auditory feature knowledge. This is consistent with our view, that concrete concepts are much dependent on features from different sensory inputs, and that atrophy in svPPA may damage a number of unimodal and multimodal anterior temporal lobe regions that support this knowledge as well as their connections with other regions of the brain. The degree of semantic impairment may vary as a function of the extent of atrophy and damage to these regions, but since temporal lobe atrophy in svPPA is typically quite extensive 
(Desgranges et al., 2007; Rogers et al., 2006), we can presume that many of these regions are affected, which leads to the multimodal semantic impairment. Recent evidence suggests that the temporopolar cortex (TPC) is not a homogeneous cortical region but is subdivided in a number of distinct subregions specialized in discrete functions (for review, see Ding et al., 2009). Based on their combined analysis of different markers, seven subregions of the TPC were identified on the lateral, dorsal and medial aspects of the temporal pole. These regions included regions thought to be involved in processing abstract and high order visual processing, high order auditory processing, polysensory visual-auditory processing, and olfactory processing. The temporal pole, the most rostral of these subregions, may also be involved in binding various perceptual inputs (including visual and auditory) to visceral responses (Ding et al., 2009). Further studies will be needed to better understand the specific roles of these subregions of the TPC.

\section{Conclusions}

In conclusion, the results of this study show a disproportionate impairment in processing concrete words vs. abstract words in svPPA. Moreover, emotional abstract word processing was between non emotional abstract word and concrete word processing in svPPA. In contrast, this pattern of results was not found in AD or in healthy aging. VBM results showed that there was an association between impaired concrete word processing and atrophy in the left ATL region. This is consistent with recent findings and suggests that this region may play a key role in multimodal sensory feature knowledge that is critical to the conceptual representations of concrete concepts.

\section{Acknowledgments}

This study was supported by the SMB's grant of the Canadian Alzheimer's Society. SJ is supported by a Chercheur-boursier senior FRQ-S award. SMB is supported by a Chercheur-boursier Junior 1 FRQ-S award. SJ, IR, and SMB are supported by the Alzheimer Society of Canada. GTV is supported by a FRQ-S postdoctoral fellowship. MM is supported by Alzheimer Society of Canada and FRQ-S doctoral awards. MAW is supported by FRQ-SC and the RQRV. RL is supported by La Fondation du CHU de Québec and La Société Alzheimer du Québec. We would like to thank all participants for taking part in this research. 


\section{References}

American Psychiatric Association. (1994). Diagnostic and Statistical Manual of Mental Disorders. Washington, DC.

Amici, S., Brambati, S. M., Wilkins, D. P., Ogar, J., Dronkers, N. L., Miller, B. L., et al. (2007). Anatomical correlates of sentence comprehension and verbal working memory in neurodegenerative disease. $J$ Neurosci, 27(23), 6282-6290.

Ashburner, J. (2007). A fast diffeomorphic image registration algorithm. Neuroimage, 38(1), 95-113.

Ashburner, J., \& Friston, K. J. (2000). Voxel-based morphometry--the methods. Neuroimage, 11(6 Pt 1), 805821.

Barense, M. D., Rogers, T. T., Bussey, T. J., Saksida, L. M., \& Graham, K. S. (2010). Influence of conceptual knowledge on visual object discrimination: insights from semantic dementia and MTL amnesia. Cereb Cortex, 20(11), 2568-2582.

Barsalou, L. W. (2008). Grounded cognition. Annu Rev Psychol, 59, 617-645.

Benton, A. L., \& Van Allen, M. W. (1968). Impairment in facial recognition in patients with cerebral disease. Cortex, 4(4), 344-IN341.

Benton, A. L., Varney, N. R., \& Hamsher, K. (1978). Visuospatial judgment: A clinical test. Archives of Neurology, 35(6), 364-367.

Binder, J. R., Desai, R. H., Graves, W. W., \& Conant, L. L. (2009). Where is the semantic system? A critical review and meta-analysis of 120 functional neuroimaging studies. Cereb Cortex, 19(12), 2767-2796.

Bonner, M. F., Ash, S., \& Grossman, M. (2010). The new classification of primary progressive aphasia into semantic, logopenic, or nonfluent/agrammatic variants. Curr Neurol Neurosci Rep, 10(6), 484-490.

Bonner, M. F., Vesely, L., Price, C., Anderson, C., Richmond, L., Farag, C., et al. (2009). Reversal of the concreteness effect in semantic dementia. Cogn Neuropsychol, 26(6), 568-579.

Brambati, S. M., Myers, D., Wilson, A., Rankin, K. P., Allison, S. C., Rosen, H. J., et al. (2006). The anatomy of category-specific object naming in neurodegenerative diseases. J Cogn Neurosci, 18(10), 1644-1653.

Brambati, S. M., Rankin, K. P., Narvid, J., Seeley, W. W., Dean, D., Rosen, H. J., et al. (2009). Atrophy progression in semantic dementia with asymmetric temporal involvement: a tensor-based morphometry study. Neurobiol Aging, 30(1), 103-111.

Breedin, S. D., Saffran, E. M., \& Coslett, H. B. (1994). Reversal of the concreteness effect in a patient with semantic dementia. Cognitive neuropsychology, 11(6), 617-660.

Bright, P., Moss, H. E., Longe, O., Stamatakis, E. A., \& Tyler, L. K. (2007). Conceptual structure modulates anteromedial temporal involvement in processing verbally presented object properties. Cereb Cortex, 17(5), 1066-1073.

Catricala, E., Della Rosa, P. A., Plebani, V., Vigliocco, G., \& Cappa, S. F. (2014). Abstract and concrete categories? Evidences from neurodegenerative diseases. Neuropsychologia, 64C, 271-281.

Corbett, F., Jefferies, E., \& Ralph, M. A. (2011). Deregulated semantic cognition follows prefrontal and temporo-parietal damage: evidence from the impact of task constraint on nonverbal object use. $J \operatorname{Cogn}$ Neurosci, 23(5), 1125-1135.

Cousins, K. A., York, C., Bauer, L., \& Grossman, M. (2016). Cognitive and anatomic double dissociation in the representation of concrete and abstract words in semantic variant and behavioral variant frontotemporal degeneration. Neuropsychologia, 84, 244-251.

Crutch, S. J. (2006). Qualitatively different semantic representations for abstract and concrete words: further evidence from the semantic reading errors of deep dyslexic patients. Neurocase, 12(2), 91-97.

Crutch, S. J., Connell, S., \& Warrington, E. K. (2009). The different representational frameworks underpinning abstract and concrete knowledge: evidence from odd-one-out judgements. $Q J$ Exp Psychol (Colchester), 62(7), 1377-1388, 1388-1390.

Crutch, S. J., Troche, J., Reilly, J., \& Ridgway, G. R. (2013). Abstract conceptual feature ratings: the role of emotion, magnitude, and other cognitive domains in the organization of abstract conceptual knowledge. Front Hum Neurosci, 7, 186.

Crutch, S. J., \& Warrington, E. K. (2005). Abstract and concrete concepts have structurally different representational frameworks. Brain, 128(Pt 3), 615-627. 
Crutch, S. J., \& Warrington, E. K. (2007). Semantic priming in deep-phonological dyslexia: contrasting effects of association and similarity upon abstract and concrete word reading. Cogn Neuropsychol, 24(6), 583-602.

Crutch, S. J., \& Warrington, E. K. (2010). The differential dependence of abstract and concrete words upon associative and similarity-based information: Complementary semantic interference and facilitation effects. Cogn Neuropsychol, 27(1), 46-71.

Desgranges, B., Matuszewski, V., Piolino, P., Chetelat, G., Mezenge, F., Landeau, B., et al. (2007). Anatomical and functional alterations in semantic dementia: a voxel-based MRI and PET study. Neurobiol Aging, 28(12), 1904-1913.

Desrochers, A. (Cartographer). (2006). OMNILEX : Une base de données informatisée sur le lexique du française contemporain

Ding, S. L., Van Hoesen, G. W., Cassell, M. D., \& Poremba, A. (2009). Parcellation of human temporal polar cortex: a combined analysis of multiple cytoarchitectonic, chemoarchitectonic, and pathological markers. $J$ Comp Neurol, 514(6), 595-623.

Fiebach, C. J., \& Friederici, A. D. (2004). Processing concrete words: fMRI evidence against a specific righthemisphere involvement. Neuropsychologia, 42(1), 62-70.

Folstein, M. F., Folstein, S. E., \& McHugh, P. R. (1975). "Mini-mental state". A practical method for grading the cognitive state of patients for the clinician. Journal of Psychiatric Research, 12, 189-198.

Franklin, S., Howard, D., \& Patterson, K. (1994). Abstract word meaning deafness. Cognitive Neuropsychology, 11, 1-34.

Franklin, S., Howard, D., \& Patterson, K. (1995). Abstract word anomia. . Cognitive Neuropsychology, 12, 549566.

Friston, K. J., Holmes, A. P., Worsley, K. J., Poline, J. B., Frith, C. D., \& Frackowiak, R. S. J. (1995). Statistical Parametric Maps in functional imaging: A general linear approach. Human Brain Mapping, 2, 189-210.

Giffard, B., Laisney, M., Desgranges, B., \& Eustache, F. (2015). An exploration of the semantic network in Alzheimer's disease: Influence of emotion and concreteness of concepts. Cortex, 69, 201-211.

Gorno-Tempini, M. L., Hillis, A. E., Weintraub, S., Kertesz, A., Mendez, M., Cappa, S. F., et al. (2011). Classification of primary progressive aphasia and its variants. Neurology, 76(11), 1006-1014.

Hodges, J. R., \& Patterson, K. (2007). Semantic dementia: a unique clinicopathological syndrome. Lancet Neurol, 6(11), 1004-1014.

Hodges, J. R., Patterson, K., Oxbury, S., \& Funnell, E. (1992). Semantic dementia. Progressive fluent aphasia with temporal lobe atrophy. Brain, 115 ( Pt 6), 1783-1806.

Hoffman, P., Jones, R. W., \& Lambon Ralph, M. A. (2013). Be concrete to be comprehended: consistent imageability effects in semantic dementia for nouns, verbs, synonyms and associates. Cortex, 49(5), 12061218.

Hoffman, P., \& Lambon Ralph, M. A. (2011). Reverse concreteness effects are not a typical feature of semantic dementia: evidence for the hub-and-spoke model of conceptual representation. Cereb Cortex, 21(9), 21032112.

Howard, D., \& Patterson, K. (1992). The Pyramids and Palm Trees Test: A test for semantic access from words and pictures. Bury St Edmunds: Thames Valley Test Company.

Jefferies, E., \& Lambon Ralph, M. A. (2006). Semantic impairment in stroke aphasia versus semantic dementia: a case-series comparison. Brain, 129(Pt 8), 2132-2147.

Jefferies, E., Patterson, K., Jones, R. W., \& Lambon Ralph, M. A. (2009). Comprehension of concrete and abstract words in semantic dementia. Neuropsychology, 23(4), 492-499.

Joanette, Y., Ska, B., \& Côté, H. (2004). Protocole Montreal d'Evaluation de la Communication (MEC): Isbergues, France.

Joubert, S., Gour, N., Guedj, E., Didic, M., Gueriot, C., Koric, L., et al. (2016). Early-onset and late-onset Alzheimer's disease are associated with distinct patterns of memory impairment. Cortex, 74, 217-232.

Kaplan, E., Goodglass, H., \& Weintraub, S. (1983). Boston Naming Test. Philadelphia.

Kousta, S. T., Vigliocco, G., Vinson, D. P., Andrews, M., \& Del Campo, E. (2011). The representation of abstract words: why emotion matters. J Exp Psychol Gen, 140(1), 14-34.

Lambon Ralph, M. A., Cipolotti, L., Manes, F., \& Patterson, K. (2010). Taking both sides: do unilateral anterior temporal lobe lesions disrupt semantic memory? Brain, 133(11), 3243-3255. 
Loiselle, M., Rouleau, I., Nguyen, D. K., Dubeau, F., Macoir, J., Whatmough, C., et al. (2012). Comprehension of concrete and abstract words in patients with selective anterior temporal lobe resection and in patients with selective amygdalo-hippocampectomy. Neuropsychologia, 50(5), 630-639.

Macoir, J. (2009). Is a plum a memory problem? Longitudinal study of the reversal of concreteness effect in a patient with semantic dementia. Neuropsychologia, 47(2), 518-535.

Martensson, F., Roll, M., Apt, P., \& Horne, M. (2011). Modeling the meaning of words: neural correlates of abstract and concrete noun processing. Acta Neurobiol Exp (Wars), 71(4), 455-478.

Martin, A. (2007). The representation of object concepts in the brain. Annu Rev Psychol, 58, 25-45.

Mathot, S., Schreij, D., \& Theeuwes, J. (2012). OpenSesame: an open-source, graphical experiment builder for the social sciences. Behav Res Methods, 44(2), 314-324.

McKhann, G. M., Knopman, D. S., Chertkow, H., Hyman, B. T., Jack, C. R., Jr., Kawas, C. H., et al. (2011). The diagnosis of dementia due to Alzheimer's disease: recommendations from the National Institute on Aging-Alzheimer's Association workgroups on diagnostic guidelines for Alzheimer's disease. Alzheimers Dement, 7(3), 263-269.

Meyers, J. E., \& Meyers, K. R. (1995). Rey complex figure test and recognition trial: Professional manual. Odessa, FL: Psychological Assessment Resources.

Morey, R. (2008). Confidence intervals from normalized data: A correction to Cousineau (2005). Tutorial in Quantitative Methods for Psychology, 4, 61-64.

New, B., Pallier, C., Brysbaert, M., \& Ferrand, L. (2004). Lexique 2 : A New French Lexical Database Behavior Research Methods, Instruments, \& Computers, 36(3), 516-524.

Osterrieth, P. A. (1944). Le test de copie d'une figure complexe; contribution à l'étude de la perception et de la mémoire. [Test of copying a complex figure; contribution to the study of perception and memory.]. Archives de Psychologie, 30, 206-356.

Paivio, A. (1986). Dual Coding Theory. In Mental Representations (pp. 53-83). New York: Oxford University Press.

Paivio, A. (1991). Dual Coding Theory: Retrospect and Current Status. Canadian Journal of Psychology, 45(3), 255-287.

Paivio, A. (2013). Dual coding theory, word abstractness, and emotion: a critical review of Kousta et al. (2011). J Exp Psychol Gen, 142(1), 282-287.

Palazova, M., Sommer, W., \& Schacht, A. (2013). Interplay of emotional valence and concreteness in word processing: an event-related potential study with verbs. Brain Lang, 125(3), 264-271.

Papagno, C., Capasso, R., \& Miceli, G. (2009). Reversed concreteness effect for nouns in a subject with semantic dementia. Neuropsychologia, 47(4), 1138-1148.

Papagno, C., Fogliata, A., Catricala, E., \& Miniussi, C. (2009). The lexical processing of abstract and concrete nouns. Brain Res, 1263, 78-86.

Patterson, K., Nestor, P. J., \& Rogers, T. T. (2007). Where do you know what you know? The representation of semantic knowledge in the human brain. Nat Rev Neurosci, 8(12), 976-987.

Peters, F., Majerus, S., De Baerdemaeker, J., Salmon, E., \& Collette, F. (2009). Impaired semantic knowledge underlies the reduced verbal short-term storage capacity in Alzheimer's disease. Neuropsychologia, 47(14), 3067-3073.

Phan, K. L., Wager, T., Taylor, S. F., \& Liberzon, I. (2002). Functional neuroanatomy of emotion: a metaanalysis of emotion activation studies in PET and fMRI. Neuroimage, 16(2), 331-348.

Pulvermuller, F. (2005). Brain mechanisms linking language and action. Nat Rev Neurosci, 6(7), 576-582.

Qualls, C. E., Bliwise, N. G., \& Stringer, A. Y. (2000). Short forms of the Benton Judgment of Line Orientation Test: development and psychometric properties. Arch Clin Neuropsychol, 15(2), 159-163.

Ralph, M. A., Jefferies, E., Patterson, K., \& Rogers, T. T. (2017). The neural and computational bases of semantic cognition. Nat Rev Neurosci, 18(1), 42-55.

Reilly, A., Cross, K., Troiani, V., \& Grossman, M. (2007). Single-word semantic judgments in semantic dementia: Do phonology and grammatical class count? Aphasiology(21), 558-569.

Reilly, J., \& Kean, J. (2007). Formal distinctiveness of high- and low-imageability nouns: analyses and theoretical implications. Cogn Sci, 31(1), 157-168.

Rey, A. (1960). Test de la Figure complexe de Rey.Unpublished manuscript, Paris. 
Rogers, T. T., Hocking, J., Noppeney, U., Mechelli, A., Gorno-Tempini, M. L., Patterson, K., et al. (2006). Anterior temporal cortex and semantic memory: reconciling findings from neuropsychology and functional imaging. Cogn Affect Behav Neurosci, 6(3), 201-213.

Rosen, H. J., Allison, S. C., Ogar, J. M., Amici, S., Rose, K., Dronkers, N., et al. (2006). Behavioral features in semantic dementia vs other forms of progressive aphasias. Neurology, 67(10), 1752-1756.

Rouleau, I., Salmon, D. P., Butters, N., Kennedy, C., \& McGuire, K. (1992). Quantitative and qualitative analyses of clock drawings in Alzheimer's and Huntington's disease. Brain Cogn, 18(1), 70-87.

Schwanenflugel, P. J., Harnishfeger, K. K., \& Stowe, R. W. (1988). Context availability and lexical decisions for abstract and concrete words. Journal of Memory and Language, 27(5), 499-520.

Schwanenflugel, P. J., \& Shoben, E. J. (1983). Differential context effects in the comprehension of abstract and concrete verbal materials. Journal of Experimental Psychology: Learning, Memory, and Cognition, 9(1), 82102.

Shallice, T., \& Cooper, R. P. (2013). Is there a semantic system for abstract words? Front Hum Neurosci, 7, 175.

Skipper-Kallal, L. M., Mirman, D., \& Olson, I. R. (2015). Converging evidence from fMRI and aphasia that the left temporoparietal cortex has an essential role in representing abstract semantic knowledge. Cortex, 69, 104120.

Strauss, E. H., Sherman, E. M. S., \& Spreen, O. (2006). A Compendium of Neuropsychological Tests. Administration norms and commentary. New York, NY: Oxford University Press.

Tombaugh, T. N. (2004). Trail Making Test A and B: normative data stratified by age and education. Arch Clin Neuropsychol, 19(2), 203-214.

Tyler, L. K., Ostrin, R. K., Cooke, M., \& Moss, H. E. (1995). Automatic access of lexical information in Broca's aphasics: against the automaticity hypothesis. Brain Lang, 48(2), 131-162.

Vallet, G. T., Simard, M., Versace, R., \& Mazza, S. (2013). The perceptual nature of audiovisual interactions for semantic knowledge in young and elderly adults. Acta Psychol (Amst), 143(3), 253-260.

Wang, J., Conder, J. A., Blitzer, D. N., \& Shinkareva, S. V. (2010). Neural representation of abstract and concrete concepts: a meta-analysis of neuroimaging studies. Hum Brain Mapp, 31(10), 1459-1468.

Warrington, E. K. (1975). The selective impairment of semantic memory. Q J Exp Psychol, 27(4), 635-657.

Wechsler, D. (1997). Wechsler Adult Intelligence Scale-III. San Antonio, TX: The Psychological Corporation.

Xiao, X., Zhao, D., Zhang, Q., \& Guo, C. Y. (2012). Retrieval of concrete words involves more contextual information than abstract words: multiple components for the concreteness effect. Brain Lang, 120(3), 251258.

Yi, H. A., Moore, P., \& Grossman, M. (2007). Reversal of the concreteness effect for verbs in patients with semantic dementia. Neuropsychology, 21(1), 9-19. 